Colquhoun, D. (2003) Challenging the tyranny of impact factors Nature, 423, 479.

Garfield, E. (1955) Citation indexes for science: a new dimension in documentation through association of ideas. Science, 122 , 108-111.

Lariviere, V. \& Gingras, Y. (2010) The impact factor's Matthew effect: a natural experiment in bibliometrics. Journal of the American Society for Information Science and Technology, 61, 424-427.

Rao, T. S. S. \& Andrade, C. (2014) Indian Journal of Psychiatry: changes in instructions to contributors. Indian Journal of Psychiatry, 56, 319-320.
Reynolds, J. C., Menegazzi, J. J. \& Yealy, D. M. (2012) Emergency medicine journal impact factor and change compared to other medical and surgical specialties. Academic Emergency Medicine, 19 , $1248-1254$

van der Wall, E. E. (2012) Journal impact factor: holy grail? Netherlands Heart Journal, 20, 385-386.

Van Diest, P. J., Holzel, H., Burnett, D., et al (2001) Impactitis: new cures for an old disease. Journal of Clinical Pathology, 54, 817-819.

Way, M. \& Ahmad, S. A. (2013) The San Francisco Declaration on Research Assessment. Journal of Cell Science, 126, 1903-1904.

SPECIAL PAPER

Formerly Executive Director of the National Mental Health Programme, Ministry of Public Health, Qatar email terence. sharkey@icloud.com

\begin{abstract}
In Qatar between 2008 and 2011, strategic developments at governmental level were beginning to reflect a more positive understanding of mental health. Under the leadership of the Ministry of Public Health, key stakeholders agreed to develop a strategy to reform the mental health system, while tackling stigma. This article discusses the development of the National Mental Health Strategy for Qatar, Changing Minds, Changing Lives, 2013-18, its implementation, and the findings from an independent impact evaluation carried out in 2015.
\end{abstract}

Qatar is a small Arab Islamic country that has undergone significant economic and demographic transition over the past half-century. Situated on a peninsula in the Persian Gulf, it now boasts the world's highest per capita gross domestic product (GDP), and the population surged from 380000 in 1986 to over 2.2 million in $2014,85 \%$ of whom are expatriates (De Bel-Air, 2014). The population has a high proportion of low-skilled single male labourers, and the male to female ratio in Qatar is 3:1. Qatari nationals and professional expatriates typically work in the governmental, financial, construction, management and education sectors.

An epidemiological shift from communicable to non-communicable or chronic diseases has taken place. Mental illnesses impact significantly on disability-adjusted life-years (DALYs) (General Secretariat of the Supreme Council of Health, 2015a,b) and quality of life.

Psychiatric services were first established in 1971 and by 1995 there were 56 in-patient beds for a population of 400000 (El-Islam, 1995). The existing provision of mental health services is still less than optimal and even now there are only 69 in-patient acute beds for a population of 2.2 million. However, significant improvements are happening, with Hamad Medical Corporation (HMC, a not-for-profit provider established by decree) providing a broad mix of in-patient and out-patient mental health services, and a new community hub has been recently established. Primary care began assessment and treatment for patients with mild to moderate mental health conditions in 2014. Additionally, major healthcare providers such as Sidra and Naufar are beginning to offer a range of high-quality services.

Between 2008 and 2011 strategic developments at governmental level were beginning to reflect a more positive understanding of the importance of mental health. There was an emerging recognition that good mental health is central to quality of life for individuals, their families, to the social and economic success of society and to the country as a whole.

Under the leadership of the Ministry of Public Health, key stakeholders agreed to develop a strategy to reform the mental health system, with a common vision of providing the best possible integrated mental health services for the people of Qatar while tackling stigma and changing attitudes towards mental illness.

This article discusses the development of the National Mental Health Strategy for Qatar, Changing Minds, Changing Lives 2013-18 (General Secretariat of the Supreme Council of Health, 2013), its implementation and the findings from an independent impact evaluation carried out in 2015 (General Secretariat of the Supreme Council of Health, 2015a,b).

\section{Why did the policy response emerge?}

In 2008 the Qatar National Health Authority (now the Ministry of Public Health) formed a National Mental Health Committee and sought technical input from the World Health Organization. The agencies worked collaboratively towards improving mental health services and this resulted in the 
development of service improvement plans which were implemented over the next 2 years.

During this period, it was concluded there was a need for a transformational change to improve mental health services in Qatar. The future system needed to be culturally appropriate and developed in consultation with service users, families and the wider community.

\section{The policy formulation process}

Senior government ministers and officials recognised that a world-class health system could not be achieved without system-wide change. A National Mental Health Implementation Committee was therefore formed to oversee policy development and implementation.

The health of the people of Qatar is at the centre of the human development pillar of the Qatar National Vision 2030 (General Secretariat for Development Planning, 2008). Goal 1 of the National Health Strategy is to create a comprehensive world-class healthcare system and it identifies mental health as a key component of a balanced care system. The new model of care seeks to create a balanced mental health system with an emphasis on shifting care from hospital to community settings. The National Mental Health Implementation Committee sought to embed mental health developments within the overall government policy framework by launching a National Mental Health Strategy in December 2013 (General Secretariat of the Supreme Council of Health, 2013).

\section{Aim of the policy response}

Ten key initiatives have been identified for an improved, comprehensive mental health system:

- raise public awareness about mental health and reduce the stigma associated with mental illness

- make mental health information resources widely available

- ensure most people can access their treatment in primary care and community settings

- develop specialist services that meet the differing needs of individuals and groups

- ensure care is individually tailored and based on treatments that work

- develop a sustainable, high-quality mental health workforce for Qatar

- provide a coordinated multi-sectoral approach to mental health policy development and planning

- enact mental health law in Qatar

- report improvements in patient care using the mental health minimum data-set

- ensure mental health research evidence translates into improvements in clinical practice and patient outcomes.

\section{Implementation and challenges}

An implementation framework with milestones and key performance indicators was planned well in advance in order to maintain the momentum. The following governance mechanisms and resources were established:

- the National Mental Health Technical Committee

- an implementation framework and project plans

- programme implementation teams

- an investment plan

- business cases for capacity-building, clinical and implementation support, and capital programmes

- external subject matter expertise.

However, some key challenges needed to be tackled in order to manage expectations. This meant being sufficiently bold and ambitious while also being realistic. One major challenge has been capacity-building in the face of a shortage of mental health professionals, limited local expertise and slow local recruitment processes.

\section{Impact of the policy}

In 2015 an independent impact evaluation (General Secretariat of the Supreme Council of Health, 2015a,b) of the strategy was undertaken based on the World Health Organization Assessment Instrument for Mental Health Systems (WHO-AIMS) (World Health Organization, 2005). The methodology comprised: a literature review; data collection; a series of meetings with officials, health professionals from primary, secondary and tertiary health services, representatives from the education sector and other relevant stakeholders; and site visits. Data collection instruments, with WHO-AIMS items and information specific to the National Mental Health Strategy, were created, adapted to the local Qatari context and piloted.

The results of the evaluation were reported in the following six domains.

\section{Policy and legislative framework}

The Qatar National Mental Health Strategy was identified as the most significant mental health policy document that the state of Qatar has ever developed. It covers most of the components recommended by the World Health Organization for national policies. A comprehensive implementation plan with initiatives and a timetable of deliverables and milestones had been formulated, and almost all deadlines have been met in a timely manner.

There was no mental health law in Qatar to safeguard the human rights of people with a mental illness but a draft law is awaiting final approval.

\section{Mental health services}

Spending on mental health in 2014 represented $0.36 \%$ of all healthcare expenditure, a figure nearly seven times lower than the median for uppermiddle-income countries. Most of the expenditures were directed towards in-patient services in the mental hospital. The Strategy clearly supported 
the transition towards more community-based care and the first community hub was opened in December 2014. It offers various mental health programmes. The average number of contacts per user in mental health out-patient centres increased over the 2-year period 2012-14, from 3.49 to 4.38. The proportion of out-patient female users was close to $50 \%$ in 2014, while the proportion of children and adolescents was $23 \%$ (a $7 \%$ increase in comparison with the percentage for 2012).

A 34-hour training programme for nurses and other professionals, developed by HMC, on recovery in mental health, represented an important contribution to capacity-building among this workforce, in line with the Strategy. This is a positive step towards more comprehensive training for all mental health specialists. Indeed, since 1993 the Department of Psychiatry at HMC has been recognised as a centre for training doctors in psychiatry by the Arab Board of Medical/Health Specialists. It was recommended that consultants should have more capacity to act as trainers and the trainer/trainee ratio should remain adequate.

\section{Mental health in primary healthcare}

Major depressive disorder and generalised anxiety disorder are the most frequent mental illnesses seen in consulting adults in Qatar's primary healthcare system, with a lifetime prevalence of $20.4 \%$ and $19.1 \%$ respectively (Ghuloum et al, 2011, 2104). However, these are likely to be underestimates because of the limited diagnostic abilities of primary care doctors at that time. Since then, all front-line family doctors have received relevant mental health training. Only $3 \%$ of undergraduate training hours for medical doctors and nurses is devoted to psychiatry, mental health and related subjects. In terms of continuing education, $56 \%$ of general practitioners and $64 \%$ of primary care family physicians received at least 2 days of training in psychiatry (or mental health more widely) during 2014, although no nurses received this type of training.

A pilot programme to treat mild to moderate common mental health conditions was launched in December 2014 in three centres. This is the first time a systematic approach has been employed using standardised screening tools, clinical guidelines for the management of depression and generalised anxiety disorders, and indications for referral to secondary care. Eventually, all primary care centres will expand the roles and functions of nurses, including more specialist roles.

\section{Human resources}

In 2014 there were, overall, 13.5 mental health professionals per 100000 population, which is less than half the median rate of upper-middle-income countries. The provision included 3.04 psychiatrists per 100000 population, 8.19 nurses, 0.89 psychologists, 0.50 occupational therapists and 0.36 social workers.

When comparing graduation rates in Qatar and the median rate in high-income countries, it is evident that, with the exception of psychiatrists, there is a training gap for mental health professionals in Qatar.

\section{Public education and links with other sectors}

In order to reduce stigma regarding mental ill health, people seeking help will have access to effective care and treatment in the most appropriate settings.

There were no organised groups of mental health service users or relatives of individuals with mental disorders, nor any mutual help (peer) groups. Five non-governmental organisations (NGOs) that carry out mental health advocacy, education and assistance activities (psychosocial support, counselling, etc.) were identified in Qatar.

The Ministry of Public Health has produced a draft action plan for the prevention of psychiatric disorder and mental health promotion, based on international best practice, local feedback and resource capacity. Alongside government efforts, numerous NGOs, private foundations and educational institutions have worked to generate public awareness and disseminate mental health information. Qualitative research focus groups and a quantitative baseline population survey were carried out to investigate local levels of awareness, attitudes and stigma, to support the development of effective campaigns and programmes.

Qatar's legislation prohibits discrimination against persons with physical, sensory, intellectual or mental disabilities, in employment, education, access to healthcare and other areas. It stipulates that $2 \%$ of jobs in government and the private sector be reserved for persons with disabilities.

All schools in Qatar have a nurse on their staff, and several schools also have a psychologist and a social worker, although currently there is no national plan or curriculum for mental health in schools. However, this was being actively pursued in 2016.

\section{Monitoring and research}

HMC regularly collects a range of data for internal performance reports and as a part of the health service provider agreements, but a more comprehensive national mental health minimum data-set was developed during 2014.

From 2011 to 2013, the annual percentage of articles on mental health listed in PubMed, in relation to all health publications on the Qatar population, was $5 \%$, and in 2014 this percentage decreased to $1.3 \%$.

In February 2016 a desktop review of the current state of mental health research governance and funding took place and this will inform the development of a priority mental health research agenda during 2016.

\section{Conclusions}

Implementation of the Strategy has been phased over 5 years $(2013-18)$ in the first instance. The implementation plan recognises the importance of sustainability and identifies the need to develop 
permanent policy and governance frameworks that will endure beyond the lifetime of the programme.

Qatar has accepted and adopted the WHO Mental Health Action Plan 2013-20 (World Health Organization, 2013) and the National Mental Health Strategy closely aligns with its key domains. The Strategy sets out a series of initiatives to create an environment that protects the dignity of people with mental health problems. So far, the provisions of common law have enabled mental health professionals to provide appropriate compulsory care. The imminent introduction of the country's first mental health law will be a landmark and it will enshrine in law the rights of patients (Abou-Saleh \& Ibrahim, 2013). El-Islam (1995) emphasised the major role of the family in decisions concerning patient admissions and follow-up care, and the role of the family will be woven into the new legal framework.

Much progress has been achieved since the launch of the Strategy but there is still a long way to go. Leaders, decision makers, professionals, patients, relatives and civil society have been able to work collaboratively towards the achievement of a shared vision where the people of Qatar can experience good mental health and well-being, supported by integrated mental health services. The country will undoubtedly achieve its vision of less stigma for those seeking help, and their access to effective care and treatment in the most appropriate settings.

\section{References}

Abou-Saleh T. \& Ibrahim N. (2013) Mental health law in Qatar. International Psychiatry, 10(4), 90. See http://www.rcpsych.ac.uk/ pdf/PUB_IPv10n4.pdf (accessed April 2016).
De Bel-Air, F. (2014) Demography, Migration and Labour Market in Qatar, GLMM-EN No. 8/2014. European University Institute and Gulf Research Centre. Available at http://cadmus.eui.eu/bitstream/ handle/1814/32431/GLMM_ExpNote_08-2014.pdf?sequence=1 (accessed May 2016)

El-Islam, M. F. (1995) Psychiatry in Qatar. Psychiatric Bulletin, 19, 779-781. Available at http://pb.rcpsych.org/content/19/12/779. full-text.pdf+html (accessed May 2016).

General Secretariat for Development Planning (2008) Qatar National Vision 2030. Available at http://www.gsdp.gov.qa/portal/ page/portal/gsdp en/qatar_national_vision/qnv_2030_document (accessed April 2016).

General Secretariat of the Supreme Council of Health (2013) Qatar National Mental Health Strategy, Changing Minds, Changing Lives 2013-2018. Available at https://www.moph.gov.qa/healthstrategies/national-mental-health-strategy (accessed April 2016).

General Secretariat of the Supreme Council of Health (2015a) Qatar National Mental Health Strategy Impact Evaluation. Supreme Council of Health.

General Secretariat of the Supreme Council of Health (2015b) Qatar Mental Health Attitudes and Awareness Baseline Measure. Supreme Council of Health.

Ghuloum, S., Bener, A. \& Abou-Saleh, M. T. (2011) Prevalence of mental disorders in adult population attending primary health care setting in Qatari population. Journal of the Pakistani Medical Association, 61(3), 216-221.

Ghuloum, S., Bener, A., Dafeeah, E. E., et al (2014) Lifetime prevalence of common mental disorders in Qatar using WHO Composite International Diagnostic Interview (WHO-CIDI). International Journal of Clinical Psychiatry and Mental Health, 2, $38-46$.

World Health Organization (2005) World Health Organization Assessment Instrument for Mental Health Systems v2.2. WHO Document Production Services. Available at http://www.who.int/ mental_health/evidence/AIMS_WHO_2_2.pdf?ua=1 (accessed April 2016).

World Health Organization (2013) Mental Health Action Plan 2013-2020. WHO Document Production Services. Available at http://www.who.int/mental_health/action_plan_2013/en/ (accessed April 2016).

SPECIAL PAPER

'South London and Maudsley NHS Foundation Trust, London, UK, email sukran83@gmail.com

${ }^{2}$ King's College London, UK, email melanie.abas@kcl.ac.uk

${ }^{3}$ London School of Hygiene and Tropical Medicine, London, UK, email cathy.zimmerman@Ishtm. ac.uk

“King's College London, UK, email louise.howard@kcl.ac.uk

${ }^{5}$ King's College London, UK, email sian.oram@kcl.ac.uk

\title{
Mental health and human trafficking: responding to survivors' needs
}

\author{
Sukran Altun, ${ }^{1}$ Melanie Abas, ${ }^{2}$ Cathy Zimmerman, ${ }^{3}$ Louise M. Howard ${ }^{4}$ and \\ Sian Oram ${ }^{5}$
}

Mental health professionals have opportunities to intervene and provide care for trafficked people. Research shows that mental health problems - including depression, anxiety and post-traumatic stress disorder - are prevalent among trafficked people, and that at least some trafficked people come into contact with secondary mental health services in England.

\section{What is human trafficking?}

Human trafficking is the recruitment and movement of people using means such as deception and coercion for the purposes of exploitation (United Nations, 2000). Men, women and children are trafficked across and within international borders for exploitation in forced sex work, domestic servitude and in a variety of industries, including fishing, agriculture and construction, as well as for forced criminal acts. Human trafficking is a global problem, with an estimated 11.7 million people exploited in the Asia-Pacific region, 3.7 million in Africa, 1.8 million in Latin America and the Caribbean, 1.6 million in central and south-eastern Europe, 1.5 million in the European Union and developed economies, and 600000 